\title{
COMPARAÇÃO DO EFEITO AGUDO DO TREINAMENTO AERÓBICO CONTÍNUO E INTERMITENTE NA GLICEMIA DE PORTADORES DE DIABETES TIPO 1
}

\author{
- um estudo de caso•
}

\author{
Valderi Abreu de Lima*, Neiva Leite**, Juliana Pereira Decimo***, Ana Claudia Kapp Titski****, \\ William Cordeiro de Souza*****, Luis Paulo Gomes Mascarenhas ${ }^{* * * * * *}$ \\ Autor correspondente: Valderi Abreu de Lima - valderiabreulima@hotmail.com \\ * Mestrando(a) em Educação Física pela Universidade Federal do Paraná \\ ** Professora de Pediatria da Universidade Federal do Paraná \\ *** Mestranda em Educação Física pela Universidade Federal do Paraná \\ **** Departamento de Pediatria da Universidade Federal do Paraná \\ ***** Núcleo de Estudos em Atividade Física, Universidade do Contestado \\ ****** Professor da Universidade do Centro Oeste
}

\section{Resumo}

Introdução: A contínua alteração glicêmica em pacientes com diabetes melittus tipo 1 acarreta em diversas complicações cardiovasculares que podem ser amenizadas pela ação do exercício físico. Objetivo: O objetivo deste estudo de caso é verificar a influência aguda do exercício aeróbio contínuo e intermitente na glicemia de diabéticos tipo 1. Métodos: Foram avaliados dois adultos praticantes de musculação há mais de três anos e portadores da diabetes melittus tipo 1. Na primeira avaliação, os indivíduos realizaram 30 minutos de exercício aeróbio contínuo em cicloergômetro, com intensidade de $60 \%$ da frequência cardíaca máxima (FCmáx). Na segunda, os avaliados foram expostos à realização de exercício aeróbio com sprints de intensidade máxima (duração de 10 segundos) intercalado com 2 minutos de exercício contínuo, até concluírem o tempo total de 30 minutos. Monitorou-se a frequência cardíaca e a glicemia capilar antes e após a realização dos exercícios. Para análise dos dados, os mesmos foram expostos em frequência (\%). Resultados: Comparando os dois protocolos de exercícios, verificou-se uma maior redução glicêmica induzida pelo exercício aeróbico contínuo $(45,5$ e $41,8 \%)$ do que aeróbico intermitente $(28,8$ e $7 \%)$, nos pacientes com diabetes tipo 1 . Observou-se um menor declínio da glicemia nos exercícios intermitentes quando comparado aos exercícios contínuos. Conclusão: A realização de exercícios aeróbios intermitentes pode ser uma estratégia na diminuição dos riscos de hipoglicemia logo após o exercício físico.

Palavras-chave: Diabete Mellitus; Glicose no Sangue; Exercício. 


\title{
COMPARISON OF THE EFFECT OF CONTINUOUS \\ AND INTERMITTENT AEROBIC TRAINING ON BLOOD GLUCOSE IN PATIENTS WITH TYPE I DIABETES
}

- a case study

\begin{abstract}
Introduction: Continuous change glucose in patients with diabetes mellitus type 1 results in several cardiovascular complications that can be softened by the action of physical exercise. Objective: The objective of this study is to verify the acute influence of continuous aerobic exercise and intermittent aerobic exercise on blood sugar diabetes type 1. Methods: We evaluated two bodybuilders adults for over three years and those of diabetes mellitus type 1. In the first assessment, subjects performed 30 minutes of continuous aerobic exercise on a cycle ergometer with intensity of $60 \%$ of maximum heart rate (MHR). On Monday, the evaluated were exposed to performing aerobic exercise with maximum intensity sprints (10 seconds duration) interspersed with two minutes of continuous exercise, to complete the total time of 30 minutes. Heart rate and blood glucose before and after the exercises was monitored. For data analysis, they were exposed frequency (\%). Results: Comparing the two exercise protocols, there was a greater reduction in blood glucose induced by continuous aerobic exercise ( 45.5 and $41.8 \%$ ) than intermittent aerobic ( 28.8 and $7 \%$ ) in patients with type 1 diabetes. There was a smaller decline of glucose in intermittent exercise compared to continuous exercise. Conclusion: This maybe a good strategy to reduce the risk of hypoglycemia after exercise.
\end{abstract}

Kepwords: Diabetes Mellitus; Blood glucose; Exercise.

\section{INTRODUÇÃO}

A contínua alteração glicêmica em pacientes com diabetes melittus tipo 1 acarreta em diversas complicações cardiovasculares que podem ser amenizadas pela ação do exercício físico.(1) Entretanto, a maioria dos pacientes com diabetes, tende a não praticar atividades físicas sistematizadas pelo receio da ocorrência da hipoglicemia, que se manifestam durante, logo após, ou horas depois da atividade realizada. $(2,3)$ Para reduzir estes riscos, a prescrição de exercícios nesta população deve ser individualizada e baseada em resultados de exames clínicos, físicos, laboratoriais e nutricionais, com acompanhamento e boa interação de uma equipe multidisciplinar. ${ }^{(4)}$
Uma alternativa recente de metodologia de treino é o exercício intermitente, que tem como principal característica a variação da intensidade, com sprints máximos e curtos, alternado com períodos de menor intensidade ou descanso. Estudos têm demonstrado o efeito positivo deste tipo de atividade na prevenção de hipoglicemia induzida pelo exercício, comparado com exercício contínuo de intensidade moderada. ${ }^{(5,6)}$ Entretanto, apesar de haver algumas pesquisas, ainda são escassos os estudos que avaliam a resposta aguda dos exercícios intermitentes, no comportamento da glicemia em pessoas com diabetes melittus tipo 1. 
Infelizmente, não há diretrizes baseadas em evidências para a participação segura em intermitente exercícios de alta intensidade, que caracteriza a maioria dos jogos de campo desportivo, ocupações trabalho manual, e jogo espontâneo. $(5,7)$ Com base nestes elementos, o objetivo deste estudo de caso é verificar a influência aguda do exercício aeróbio contínuo e intermitente na glicemia de diabéticos tipo 1.

\section{MÉTODO}

Participaram do estudo dois adultos praticantes de musculação há mais de três anos e portadores da diabetes melittus tipo 1. Esses indivíduos fazem uso de insulina NPH e regular, contudo não usam nenhum outro medicamento.

Os avaliados preencheram um termo de consentimento livre e esclarecido, após a explicação dos objetivos e dos procedimentos metodológicos do estudo. Este trabalho foi aprovado pelo Comitê de Ética da Universidade do Contestado - UnC (número do Parecer: 03682912.6.0000.0117).

Antes da realização dos testes físicos, os dois sujeitos passaram por uma avaliação nutricional. Os mesmos foram orientados a consumir uma refeição composta por um sanduíche de pão integral com queijo branco, uma xícara de café com leite e uma maçã, 60 minutos antes dos testes (contínuo e intermitente), como demonstrado no Quadro 1.

Quadro 1 - Descrição dos macronutrientes ingeridos 60 minutos antes dos testes. (\%VD) Valores Diários de referência com base em uma dieta de $2.500 \mathrm{Kcal}$

\begin{tabular}{|c|c|c|c|c|c|}
\hline REFEIÇão & GRAMAS & KCAL & PROTEÍNA \%VD & CARBOIDRATOS \%VD & LIPÍDIOS \%VD \\
\hline PÃO & 50 & 6 & 6,4 & 4,42 & 2,0 \\
\hline QUEIJO & 30 & 10,1 & 2,6 & 9,28 & 4,46 \\
\hline MAÇã & 100 & 3,2 & 3,7 & 0 & 0,32 \\
\hline LEITE & 50 & 3,3 & 5,3 & 2,8 & 18,72 \\
\hline CAFÉ & 125 & 0 & 0 & 0 & 0 \\
\hline
\end{tabular}

Os testes físicos foram realizados em cicloergômetro, com uma semana de intervalo entre eles. $\mathrm{Na}$ primeira avaliação, os indivíduos realizaram 30 minutos de exercício aeróbio contínuo em cicloergômetro, com intensidade de $60 \%$ da frequência cardíaca máxima (FCmáx), previamente calculada pela fórmula de Tanaka. ${ }^{(8)}$

Uma semana depois, os sujeitos retornaram para realizar a segunda avaliação. Nesta, os avaliados foram expostos à realização de exercício aeróbio, com 10 segundos de sprints de intensidade máxima, intercalado com 2 minutos de exercício contínuo, até concluírem o tempo total de 30 minutos.

Durante o exercício, monitorou-se a frequência cardíaca, com frequencímetro da marca Oregon
HR 102. Da mesma forma, a glicemia capilar foi avaliada antes e após a realização dos exercícios por meio de um glicosímetro marca G-TECH free. Para segurança dos avaliados durante os testes estavam disponíveis fontes de carboidratos em caso de ocorrência de hipoglicemias induzidas pelo exercício.

A análise dos dados foi realizada por meio de estatística descritiva e a variação entre as avaliações foi realizada através da variação percentual (\%).

\section{RESULTADOS}

Participaram do estudo de caso dois indivíduos. $\mathrm{O}$ paciente $A$ é do sexo masculino tem 30 anos de idade e diagnostico de diabetes melittus tipo 1 há 
7 anos. Já o paciente $B$ também tem 30 anos e o tempo de diagnostico é 8 anos. Na Tabela 1 são apresentados os dados de massa corporal ( $\mathrm{kg}$ ), estatura $(\mathrm{cm})$, IMC $\left(\mathrm{kg} / \mathrm{m}^{2}\right)$ e hemoglobina glicada.

Tabela 1 - Caracterização da amostra

\begin{tabular}{|c|c|c|c|c|c|c|}
\hline & $\begin{array}{l}\text { IDADE } \\
\text { (ANOS) }\end{array}$ & $\begin{array}{c}\text { MASSA } \\
\text { CORPORAL } \\
\text { (KG) }\end{array}$ & $\begin{array}{c}\text { ESTATURA } \\
\text { (CM) }\end{array}$ & $\begin{array}{l}\text { IMC } \\
(K G / M \cdot)\end{array}$ & $\begin{array}{c}\text { HeMOGLOBINA } \\
\text { GLICADA } \\
\text { HBAIC }\end{array}$ & $\begin{array}{c}\text { TEMPO DE } \\
\text { DIAGNÓSTICO } \\
\text { (ANOS) }\end{array}$ \\
\hline $\begin{array}{c}\text { SUJEITO } \\
1\end{array}$ & 30 & 72 & 1,70 & 24,91 & $7,2 \%$ & 7 \\
\hline $\begin{array}{c}\text { SUJEITO } \\
2\end{array}$ & 30 & 68 & 1,68 & 24,11 & 7,०\% & 8 \\
\hline
\end{tabular}

$\mathrm{Na}$ Tabela 2 estão expostos os valores da glicetínuo como para o intermitente. mia pré e pós-exercício, tanto para o exercício con-

Tabela 2 - Valores de glicemia pré e pós-exercício contínuo e intermitente

\begin{tabular}{ccccccc}
\hline & $\begin{array}{c}\text { PRÉ- } \\
\text { EXERCícIO } \\
\text { CONTINUO } \\
(\mathrm{MG} / \mathrm{DL})\end{array}$ & $\begin{array}{c}\text { Pós- } \\
\text { EXERCícIO } \\
\text { CONTINUO } \\
(\mathrm{MG} / \mathrm{DL})\end{array}$ & $\begin{array}{c}\text { \% DE } \\
\text { VARIAÇÃo }\end{array}$ & $\begin{array}{c}\text { PRÉ- } \\
\text { EXERCícIO } \\
\text { INTERMITENTE } \\
\text { (MG/DL) }\end{array}$ & $\begin{array}{c}\text { Pós- } \\
\text { EXERCícIO } \\
\text { INTERMITENTE } \\
\text { (MG/DL) }\end{array}$ & $\begin{array}{c}\% \text { DE } \\
\text { VARIAÇÃo }\end{array}$ \\
\hline $\begin{array}{c}\text { SUJEITO } \\
\mathbf{1}\end{array}$ & 222 & 121 & $-45,5 \%$ & 108 & 77 & $-28,8 \%$ \\
\hline $\begin{array}{c}\text { SUJEITO } \\
\mathbf{2}\end{array}$ & 122 & 71 & $-41,8 \%$ & 269 & 252 & $-7,0 \%$ \\
\hline
\end{tabular}

\section{DISCUSSÕES}

Neste estudo, o objetivo principal foi verificar a influência aguda do exercício aeróbio contínuo e exercício aeróbio intermitente na glicemia de diabéticos tipo 1. Os dois casos apresentados relatam diferença no comportamento glicêmico de acordo com o tipo de exercício realizado (continuo ou intermitente).

Como observado por Rosenbloom, Silverstein, Amemiya, Zeitler e Klingensmith( ${ }^{(9)}$ o controle metabólico do diabetes é representado pelos valores de hemoglobina glicada ( $\mathrm{HbArc}$ ). Neste trabalho, os dois sujeitos apresentam este controle, pois os valores são inferiores a 7,5\% (7,2\% vs 7\%). Esse re- sultado pode estar associado ao fato dos mesmos serem ativos fisicamente por um longo período, corroborando com a literatura, ${ }^{(10)}$ que afirma que a prática regular de atividades físicas está associada a um melhor controle metabólico evidenciado por uma hemoglobina glicada (Arc) menor. ${ }^{(11)}$

Em relação ao exercício físico, este estudo demonstrou que ambas as metodologias, tanto a contínua como intermitente, induzem uma redução da glicemia capilar (Tabela 2). Esta redução já era esperada, uma vez que o exercício físico induz a um aumento no consumo da glicose pelo músculo esquelético em até 20 vezes em comparação a valores de repouso, representando uma elevação de 25 a $40 \%$ do total do substrato oxidado pelo organismo. ${ }^{(4)}$ 
Ao comparar os dois protocolos de exercícios, identifica-se uma redução mais acentuada da glicemia induzida pelos exercícios aeróbios contínuos $(45,5 \%$ e $41,8 \%)$ do que aeróbios intermitentes $(28,8 \%$ e $7 \%)$. Em estudo com desenho semelhante, com 30 minutos de exercícios contínuos em cicloergômetro a $40 \%$ do $\mathrm{VO}_{2}$ pico e sprints, observou-se um declínio maior da glicemia no exercício continuo do que no intermitente, ${ }^{(13)}$ concordando com os resultados do presente trabalho. Além do estudo mencionado, resultados parecidos foram encontrados por Guelfi, Jones e Fournier(5) que observaram um maior decréscimo na glicemia com exercícios contínuos quando comparados com exercícios intermitentes. Os autores perceberam que além deste resultado, os níveis de lactato sanguíneo, hormônio $\mathrm{GH}$, catecolaminas se encontravam mais elevados após exercício intermitente. Fato este que pode explicar a menor redução glicêmica durante o exercício intermitente, uma vez que esses hormônios estimulam o aumento da produção de glicose hepática via gliconeogenese, evitando assim, o declínio dos níveis plasmáticos de glicose durante o exercício.

Iscoe e Riddell(14) realizaram estudo com 11 atletas diabéticos que realizaram duas metodologias de treino. Na primeira, os sujeitos se exercitaram por 45 minutos a $40 \%$ do $\mathrm{VO}_{2}$ máx intercalado com 9 sprints de máxima intensidade por 15 segundos, já na segunda, realizaram de forma continua na mesma intensidade e duração, e não observaram diferenças entre as duas metodologias de exercício e uma redução na glicemia plasmática de 50,6\% em ambas. Os autores contradizem os achados pós-exercício, contudo sugerem que a atividade intermitente parece ser um agente protetor da hipoglicemia noturna.

Nesta mesma linha, outro estudo demonstrou uma boa resposta no controle glicêmico de diabéticos submetido a sessões de treinamento em circuito intercalando exercícios de musculação e aeróbio, observando um redução da hemoglobina glicada após 3 meses de treinamento ${ }^{(12)}$ e que exercícios de cunho anaeróbicos posteriores aos aeró- bicos induzem a uma menor redução da glicemia plasmática em pacientes diabéticos tipo 1.

O presente estudo apresenta limitações devido ao numero amostral e pouco controle ou avaliação de variáveis intervenientes como insulinoterapia, níveis hormonais e hábitos alimentares. Futuras pesquisas devem ser realizadas com um maior número de participantes, controle mais rigoroso das variáveis intervenientes, bem como de forma longitudinal com o intuito de melhorar o entendimento das respostas metabólicas de diabéticos melitus tipo 1 que praticam atividades esportivas.

\section{CONSIDERAÇÕES FINAIS}

Este estudo demonstrou que a realização de $30 \mathrm{mi}$ nutos de exercícios aeróbios em cicloergômetro a $60 \%$ da FCM e ou intercalado com sprints máximos de 10 segundos promovem redução dos níveis glicêmicos avaliados. Este declínio se mostrou menor amplitude nos exercícios intermitentes, o que pode ser uma estratégia interessante na diminuição do risco de hipoglicemias após o exercício.

\section{REFERÊNCIAS}

1. Rosa JS, Flores RL, Oliver SR, Pontello AM, Zaldivar FP, Galasseti PR. Resting and exercise-induced IL-6 levels in children with Type 1 diabetes reflect hiperglycemic profiles during the previous 3 days. J. appl. physiol. 2010;108(2):334-42.

2. The Diabetes Control and Complications Trial Research Group (DCCT). The effect of Intensive Treatment of Diabetes on the Development and Progression of Long-term Complications in Insulin-dependent Diabetes Mellitus. N. Engl. j. med. 1993; 329(14):977-86.

3. Sociedade Brasileira de Diabetes. Como prescrever o exercício no tratamento do diabetes Mellitus. Grupo Editorial Nacional. 2013-2014.

4. Martins DM, Duarte FMS. Efeitos do exercício físico sobre o comportamento da glicemia em 
indivíduos diabéticos. Rev. bras. ativ. fís. saúde. 1998;3(3):32-44.

5. Guelfi KJ, Jones TW, Fournier PA. The Decline in Blood Glucose levels Is Less With Intermittent High-Intensity Compared With Moderate Exercise in Individuals With Type 1 Diabetes. Diabetes Care. 2005;28(6):1289-94.

6. Guelfi KJ, Ratnam N, Smythe GA, Jones TW, Fournier PA. Effect of intermittent high-intensity compared with continuous moderate exercise on glucose production and utilization in individuals with type 1 diabetes. Am. j. physiol. endocrinol. metab. 2007;392(3):865-70.

7. Harmer AR, Chisholm DJ, McKenna MJ, Morris NR, Thom JM, Bennett, G. et al. High-intensity training improves plasma glucose and acidbase regulation during intermittent maximal exercise in type 1 diabetes. Diabetes Care. 2007;30(5):1269-71.

8. Tanaka H, Monahan KD, Seals DR. Agepredicted maximal heart rate revisited. J. Am. Coll. Cardiol. 2011;37(1):153-56.

9. Rosenbloom AL, Silverstein JH, Amemiya $\mathrm{S}$, Zeitler P, Klingensmith GJ. Type 2 diabetes in the child and adolescent. Pediatr. diabetes. 2009;10(Suppl. 12):17-32.
10. Sociedade Brasileira de Diabetes. Alvos no controle clínico e metabólico de crianças e adolescentes com diabetes mellitus tipol. Grupo Editorial Nacional. 2013-2O14.

11. Valerio G, Spagnuolo MI, Lombardi F, Spadaro R, Siano M, Franzese A. Physical activity and sports participation in children and adolescents with type 1 diabetes mellitus. Nutr. metab. cardiovasc. dis. 2007;17(5):376-82.

12. Lima VA, Mascarenhas LPG, Grzelczak MT, França SN. A influência do treinamento resistido e aeróbio em forma de circuit training no controle glicêmico do diabetes tipo 1: Estudo de caso. Rev. Uniandrade. 2012;13(3):248-57.

13. Guelfi KJ, Jones TW, Fournier PA. Intermittent High-Intensity Exercise Does Not Increase the Risk of Early Postexercise Hypoglycemia in Individuals With Type 1 Diabetes. Diabetes Care. 2005;28(2):416-18.

14. Iscoe KE, Riddell MC. Continuous moderateintensity exercise with or without intermittent high-intensity work: Effects on acute and late glycaemia in athletes with Type 1 diabetes mellitus. Diabet. med. 2011;28(7):824-32. 